\title{
COMMENTARY
}

\section{Cannabinoid Receptor 2 Activation}

\section{A Means to Prevent Monocyte-Endothelium Engagement}

\author{
Shilpa J. Buch
}

\begin{abstract}
From the Department of Pharmacology and Experimental Neuroscience, University of Nebraska Medical Center, Omaha, Nebraska
CME Accreditation Statement: This activity ("ASIP 2013 AJP CME Program in Pathogenesis") has been planned and implemented in accordance with the Essential Areas and policies of the Accreditation Council for Continuing Medical Education (ACCME) through the joint sponsorship of the American Society for Clinical Pathology (ASCP) and the American Society for Investigative Pathology (ASIP). ASCP is accredited by the ACCME to provide continuing medical education for physicians.
\end{abstract}

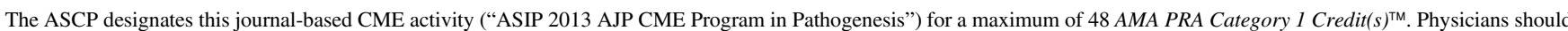
only claim credit commensurate with the extent of their participation in the activity.

CME Disclosures: The authors of this article and the planning committee members and staff have no relevant financial relationships with commercial interests to disclose.

The development and maintenance of chronic inflammation appear to be underlying causes of numerous human diseases such as atherosclerosis, obesity, diabetes, arthritis, chronic hepatitis, bone disorders, and neurodegeneration (including Alzheimer disease, multiple sclerosis, and chronic infections of the central nervous system like HIV-1). ${ }^{1-4}$ In all of these diseases, tissue infiltration by monocytes differentiating into macrophages plays a critical role, promoting development of atherosclerotic plaques, liver fibrosis, microglial accumulation around $\beta$-amyloid plaques in the brain, and microphage/microglial nodules in HIV-1 encephalitis, to name a few. Pro-inflammatory factors secreted by these cells are the driving force of organ specific injuries like neuronal death, hepatocyte demise and liver fibrosis, destruction of cartilage, or desensitization of insulin receptors. Inflammatory cell engagement of the endothelium leads to endothelial barrier compromise, further exacerbating tissue damage. This is especially important for the blood-brain barrier (BBB), which guards the unique microenvironment in the central nervous system and assures proper function of neuronal circuits. ${ }^{5}$ Despite a significant body of research on this subject, clear understanding of mechanisms, and therefore protective strategies, preventing them are missing. The work published in this issue by Rom et $\mathrm{al}^{6}$ addresses an important and an understudied problem of the selective effects of cannabinoid receptor (CB) 2 stimulation in leukocytes versus endothelial cells.

In this regard, the cannabinoid system could offer mechanistic insight into the development, propagation, and resolution of chronic inflammation. Two types of cannabinoid receptors have been discovered and are relatively conserved in different species. CB1 was originally detected mainly in the central nervous system on certain types of neurons. ${ }^{7}$ Its stimulation mediates the psychoactive effects of marijuana. CB2 is predominantly expressed on immune cells ( $\mathrm{T}$ and B lymphocytes, monocytes, macrophages, and microglia) and more recently has been found on endothelial cells. B lymphocytes, monocytes, and macrophages are the cell types that most highly express CB2 receptors in both rodents and humans. ${ }^{8}$

Endocannabinoids produced by numerous cell types in the body react with both $\mathrm{CB} 1$ and $\mathrm{CB} 2$, thereby regulating a number of important functions. ${ }^{9}$ One of the potential roles of CB2 activation is modulation of immune and inflammatory responses. The protective anti-inflammatory effects of CB2 stimulation by highly selective agonists have been documented in animal models for atherosclerosis, ${ }^{10}$ chronic neuroinflammation (multiple sclerosis), ${ }^{11}$ Alzheimer disease, ${ }^{12}$ liver and kidney injury, ${ }^{13}$ and arthritis. ${ }^{14}$ All factors implicated in inflammation-associated neurodegeneration-like secretion of pro-inflammatory factors, excitotoxicity, oxidative stress, ${ }^{15}$ and impairment of the $\mathrm{BBB}^{3}$ - could be mitigated by $\mathrm{CB} 2$ stimulation. Indeed, $\mathrm{CB} 2$ agonists are able to reduce leukocyte migration ${ }^{16}$ and cytokine production by immune cells $^{17}$ and protect the endothelial barrier by decreasing

Accepted for publication August 21, 2013.

Address correspondence to Shilpa Buch, Ph.D., Department of Pharmacology \& Experimental Neuroscience, 985930 University of Nebraska Medical Center, Omaha, NE 68198. E-mail: sbuch@unmc.edu 
leukocyte-brain endothelial engagement. ${ }^{3}$ Furthermore, activation of $\mathrm{CB} 2$ provides immunomodulation in response to pathogens or transplantation of solid organs. All these data point to the potential clinical benefits of selective CB2 agonists that are devoid of the psychoactive effects of CB1 activation. Yet, such compounds are not being used in clinical practice.

End-organ injury in chronic inflammation is driven by immune cell infiltration. It is known that CB2 agonists limit inflammatory cell migration at the site of acute and chronic injury such as ischemia and reperfusion injury, atherosclerosis, multiple sclerosis, hepatitis, cellular rejection of transplanted solid organs, etc, in rodent models (lymphocytes, neutrophils, monocytes, and macrophages). ${ }^{9}$ However, it is not clear whether endothelial cells, immune and inflammatory cells, or both are responsible for mediating these beneficial properties, and the relative contribution of each cell type is yet unknown. To address this question, Rom et $\mathrm{al}^{6}$ used in vivo imaging and adoptive transfer of leukocytes treated with CB2 agonists ex vivo in an animal model of systemic inflammation on lipopolysaccharide administration. They demonstrated a $>90 \%$ decrease in leukocyte adhesion to brain endothelium. Furthermore, they demonstrated a 50\% increase in adhesion of leukocytes from CB2 knockout animals when they were transferred to wild-type mice injected with lipopolysaccharide. For the first time, they demonstrated the selective effects of CB2 stimulation in leukocytes versus endothelial cells.

Interactions between endothelium and inflammatory cells are critically important in the initiation and propagation of inflammatory injury. Leukocyte rolling, adhesion, and eventual migration across endothelium are tightly regulated by expression of adhesion molecules on endothelium (intercellular adhesion molecule, vascular cell adhesion molecule 1 , and others) and their respective ligands [lymphocyte function-associated antigen 1, very late antigen 4 (VLA-4), etc] on lymphocytes, monocytes, and other inflammatory cells. Despite indirect evidence from animal studies, there is very limited data on how CB2 signaling mediates its antiinflammatory effects in monocytes and macrophages, particularly in human cells. Rom et $\mathrm{al}^{6}$ used highly relevant human cellular systems, such as primary brain microvascular endothelial cells and primary human monocytes, to selectively activate CB2 in human monocytes. They showed decreased adhesion to and migration of monocytes across activated brain endothelial monolayers, and also established that inhibition of small GTPases (RhoA and Rac1) was responsible for these effects. Furthermore, these outcomes were associated with specific cytoskeletal changes, diminished lamellipodia formation, and down-regulation of the active form of VLA-4 (but not total VLA-4 expression). These results provide novel mechanistic insights on the protective effects of CB2. Although some effects such as RhoA inhibition and diminished migration have been documented before, ${ }^{18}$ none of the previous studies have addressed anti-inflammatory effects of CB2 activation in monocytes in such a comprehensive fashion.

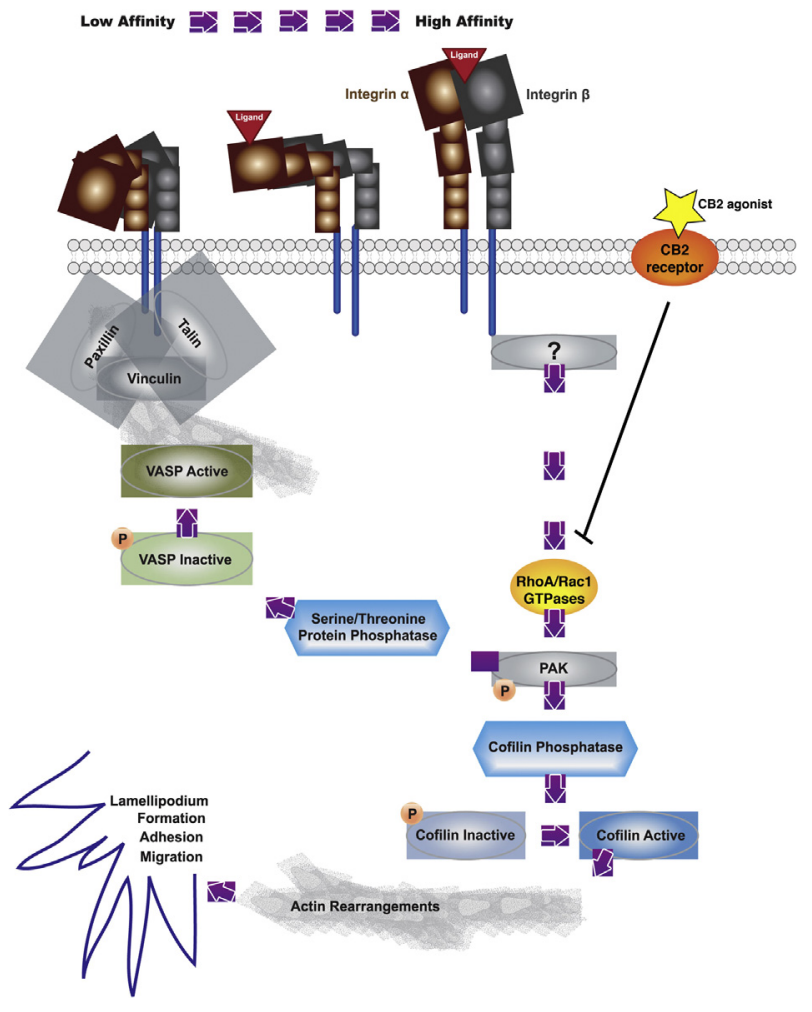

Figure 1 Cannabinoid receptor (CB) 2 stimulation in monocytes: Signaling pathways. CB2 activation in monocytes leads to $i$ ) the inhibition of small GTPases, RhoA, and Rac1, ii) cytoskeleton rearrangement via augmented levels of inhibitory sites of the actin binding proteins, cofilin and VASP, upstream regulators of conformational integrin changes, iii) prevention of lamellipodia formation (key factors in monocyte migration), and iv) down-regulation of expression of integrin VLA-4 active form (mediating adhesion to vascular cell adhesion molecule 1 expressing endothelial cells). The combination of these events results in decreased monocyte adhesion to and migration across brain endothelial cells and protects blood-brain barrier.

Nevertheless, the study has some limitations. Specifically, in vivo imaging of transferred cells has been limited to 1 hour and BBB permeability was not assessed. On balance, the use of real-time measures of barrier function in primary brain microvascular endothelial cells in this study demonstrated the protective effect of CB2 activation in monocytes. It is worth mentioning that the same group has also demonstrated positive effects of CB2 stimulation in brain endothelium resulting in diminution of the pro-inflammatory phenotype of human brain endothelial cells and shielding of the BBB under inflammatory and physiological conditions confirming these in vitro assays by intravital microscopy. ${ }^{3}$ Figure 1 summarizes the signaling pathways involved in CB2 stimulation in monocytes. Taken together, it appears that CB2 stimulation could be a powerful tool in the treatment of inflammation and immunomodulation. It is critical to further develop a number of these compounds and thoroughly investigate their long term in vivo effects.

An important question that remains to be answered is what actual physiological role the endocannabinoid system plays in inflammatory response. ${ }^{19}$ It seems to be up-regulated in 
inflammation as shown by increased expression of CB2 in Alzheimer disease, multiple sclerosis, and HIV encephalitis with enhanced expression of its synthesizing and degrading enzymes. ${ }^{19,20}$ Furthermore, the endocannabinoid system may be involved in inflammation resolution. This point is critical for chronic inflammation, where exaggerated and poorly controlled inflammatory reactions propagate tissue damage. Additional confirmation comes from a recent study indicating that chronic hepatitis $\mathrm{C}$ patients with polymorphism in CB2 are prone to severe necrotizing inflammation. ${ }^{21}$

As mentioned above, there is emerging clinical evidence for the importance of targeting monocytes and macrophages in chronic inflammatory diseases and on the therapeutic potential of selective CB2 agonists in large number of conditions. It is not clear what role CB1 plays in inflammation. There are seemingly opposing regulatory roles of $\mathrm{CB} 1$ and $\mathrm{CB} 2$ in monocytes and macrophages. ${ }^{22}$ Several studies have shown that CB1 antagonists are protective in atherosclerosis models, decreasing macrophage inflammatory response, ${ }^{23}$ reactive oxygen species generation, and cytokine production. ${ }^{24,25}$ It will be very important to understand these signaling events to design better therapies. These observations recommend the rationale of combining peripherally restricted CB2 agonists with peripherally restricted CB1 antagonists.

\section{References}

1. Libby P, Lichtman AH, Hansson GK: Immune effector mechanisms implicated in atherosclerosis: from mice to humans. Immunity 2013, 38:1092-1104

2. Han JM, Levings MK: Immune regulation in obesity-associated adipose inflammation. J Immunol 2013, 191:527-532

3. Ramirez SH, Hasko J, Skuba A, Fan S, Dykstra H, McCormick R, Reichenbach N, Krizbai I, Mahadevan A, Zhang M, Tuma R, Son YJ, Persidsky Y: Activation of cannabinoid receptor 2 attenuates leukocyteendothelial cell interactions and blood-brain barrier dysfunction under inflammatory conditions. J Neurosci 2012, 32:4004-4016

4. Di Marco E, Gray SP, Jandeleit-Dahm K: Diabetes alters activation and repression of pro- and anti-inflammatory signaling pathways in the vasculature. Front Endocrinol (Lausanne) 2013, 4:68

5. Persidsky Y, Ramirez SH, Haorah J, Kanmogne GD: Blood-brain barrier: structural components and function under physiologic and pathologic conditions. J Neuroimmune Pharmacol 2006, 1:223-236

6. Rom S, Zuluaga-Ramirez V, Dykstra H, Reichenbach NL, Pacher P, Persidsky Y: Selective activation of cannabinoid receptor 2 in leukocytes suppresses their engagement of the brain endothelium and protects the blood brain barrier (BBB). Am J Pathol 2013, 183:1548-1558

7. Ashton $\mathrm{CH}$, Moore PB: Endocannabinoid system dysfunction in mood and related disorders. Acta Psychiatr Scand 2011, 124:250-261

8. Anday JK, Mercier RW: Gene ancestry of the cannabinoid receptor family. Pharmacol Res 2005, 52:463-466

9. Rom S, Persidsky Y: Cannabinoid receptor 2: potential role in immunomodulation and neuroinflammation. J Neuroimmune Pharmacol 2013, 8:608-620

10. Rajesh M, Mukhopadhyay P, Batkai S, Patel V, Saito K, Matsumoto S, Kashiwaya Y, Horvath B, Mukhopadhyay B, Becker L, Hasko G,
Liaudet L, Wink DA, Veves A, Mechoulam R, Pacher P: Cannabidiol attenuates cardiac dysfunction, oxidative stress, fibrosis, and inflammatory and cell death signaling pathways in diabetic cardiomyopathy. J Am Coll Cardiol 2010, 56:2115-2125

11. Benito C, Romero JP, Tolon RM, Clemente D, Docagne F, Hillard CJ, Guaza C, Romero J: Cannabinoid CB1 and CB2 receptors and fatty acid amide hydrolase are specific markers of plaque cell subtypes in human multiple sclerosis. J Neurosci 2007, 27:2396-2402

12. Ramirez BG, Blazquez C, Gomez del Pulgar T, Guzman M, de Ceballos ML: Prevention of Alzheimer's disease pathology by cannabinoids: neuroprotection mediated by blockade of microglial activation. J Neurosci 2005, 25:1904-1913

13. Mukhopadhyay $\mathrm{P}$, Rajesh M, Horvath B, Batkai S, Park O, Tanchian G, Gao RY, Patel V, Wink DA, Liaudet L, Hasko G, Mechoulam R, Pacher P: Cannabidiol protects against hepatic ischemia/reperfusion injury by attenuating inflammatory signaling and response, oxidative/nitrative stress, and cell death. Free Radic Biol Med 2011, 50:1368-1381

14. Mbvundula EC, Bunning RA, Rainsford KD: Arthritis and cannabinoids: hU-210 and Win-55,212-2 prevent IL-1alpha-induced matrix degradation in bovine articular chondrocytes in-vitro. J Pharm Pharmacol 2006, 58:351-358

15. Downer EJ, Clifford E, Gran B, Nel HJ, Fallon PG, Moynagh PN: Identification of the synthetic cannabinoid R(+)WIN55,212-2 as a novel regulator of IFN regulatory factor 3 activation and IFN-beta expression: relevance to therapeutic effects in models of multiple sclerosis. J Biol Chem 2011, 286:10316-10328

16. Miller AM, Stella N: CB2 receptor-mediated migration of immune cells: it can go either way. Br J Pharmacol 2008, 153:299-308

17. Facchinetti F, Del Giudice E, Furegato S, Passarotto M, Leon A: Cannabinoids ablate release of TNFalpha in rat microglial cells stimulated with lypopolysaccharide. Glia 2003, 41:161-168

18. Montecucco F, Burger F, Mach F, Steffens S: CB2 cannabinoid receptor agonist JWH-015 modulates human monocyte migration through defined intracellular signaling pathways. Am J Physiol Heart Circ Physiol 2008, 294:H1145-H1155

19. Pacher P, Mechoulam R: Is lipid signaling through cannabinoid 2 receptors part of a protective system? Prog Lipid Res 2011, 50:193-211

20. Benito C, Kim WK, Chavarria I, Hillard CJ, Mackie K, Tolon RM, Williams K, Romero J: A glial endogenous cannabinoid system is upregulated in the brains of macaques with simian immunodeficiency virus-induced encephalitis. J Neurosci 2005, 25:2530-2536

21. Coppola N, Zampino R, Bellini G, Macera M, Marrone A, Pisaturo M, Boemio A, Nobili B, Pasquale G, Maione S, Adinolfi LE, Perrone L, Sagnelli E, Del Giudice EM, Rossi F: Association between a polymorphism in cannabinoid receptor 2 and severe necroinflammation in patients with chronic hepatitis C. Clin Gastroenterol Hepatol 2013. http://dx.doi.org/10.1016/j.cgh.2013.05.008. [Epub ahead of print]

22. Pacher P, Kunos G: Modulating the endocannabinoid system in human health and disease-successes and failures. FEBS J 2013, 280:1918-1943

23. Han KH, Lim S, Ryu J, Lee CW, Kim Y, Kang JH, Kang SS, Ahn YK, Park CS, Kim JJ: CB1 and CB2 cannabinoid receptors differentially regulate the production of reactive oxygen species by macrophages. Cardiovasc Res 2009, 84:378-386

24. Caraceni P, Pertosa AM, Giannone F, Domenicali M, Grattagliano I, Principe A, Mastroleo C, Perrelli MG, Cutrin J, Trevisani F, Croci T, Bernardi M: Antagonism of the cannabinoid CB-1 receptor protects rat liver against ischaemia-reperfusion injury complicated by endotoxaemia. Gut 2009, 58:1135-1143

25. Mnich SJ, Hiebsch RR, Huff RM, Muthian S: Anti-inflammatory properties of CB1-receptor antagonist involves beta2 adrenoceptors. J Pharmacol Exp Ther 2010, 333:445-453 